\title{
Experimental Study on the Unsteady Characteristics and the Impact Performance of a High-Pressure Submerged Cavitation Jet
}

\author{
Yongfei Yang, ${ }^{1}$ Wei Li $\left(\mathbb{D},{ }^{1}\right.$ Weidong Shi ${ }^{2},{ }^{2}$ Ling Zhou, ${ }^{1}$ and Wenquan Zhang ${ }^{1}$ \\ ${ }^{1}$ Research Center of Fluid Machinery Engineering and Technology, Jiangsu University, Zhenjiang 212013, China \\ ${ }^{2}$ College of Mechanical Engineering, Nantong University, Nantong 226019, China
}

Correspondence should be addressed to Wei Li; lwjiangda@ujs.edu.cn and Weidong Shi; wdshi@ujs.edu.cn

Received 11 October 2019; Revised 15 March 2020; Accepted 25 May 2020; Published 16 June 2020

Academic Editor: Wahyu Caesarendra

Copyright ( 2020 Yongfei Yang et al. This is an open access article distributed under the Creative Commons Attribution License, which permits unrestricted use, distribution, and reproduction in any medium, provided the original work is properly cited.

High-pressure submerged cavitation jet is widely used in the fields of material peening, petroleum drilling, and ocean engineering. The impact performance of the jet with intensive cavitation is related to the factors such as working condition and the nozzle geometry. To reveal the relationship between the nozzle divergent angle and the jet pressure on the unsteady characteristics of the jet, high-speed photography with frame rate of $20000 \mathrm{fps}$ is used to record the image of the cavitation clouds. Grayscale analysis algorithm developed in MATLAB is used to study the effects of injecting condition on the special structure, unsteady characteristics, and shedding frequency of the cavitation bubbles. The impact load characteristics of the cavitation jet with different cavitation numbers and stand-off distances are recorded using a high-response pressure transducer. It is found that the cavitation number is the main factor affecting the cavitation morphology of the submerged jet. The lower the cavitation number is, the more intense the cavitation occurs. The outlet divergent angle of the convergent-divergent nozzle also has a significant influence on the development of the cavitation clouds. In the three nozzles with the outlet divergent angles of $40^{\circ}, 80^{\circ}$, and $120^{\circ}$, the highest bubble concentration is formed usinga nozzle with a divergent angle of $40^{\circ}$, but the high-concentration cavitating bubbles are only distributed in a very small range of the nozzle outlet. The cavities generated by using the nozzle with a divergent angle of $80^{\circ}$ can achieve good results in terms of concentration and distribution range, while the nozzle with divergent angle of $120^{\circ}$ has lower cavitation performance due to the lack of the constraint at the outlet which intensifies the shear stress of the jet. According to the result of frame difference method (FDM) analysis, the jet cavitation is mainly formed in the vortex structure generated by the shearing layer at the nozzle exit, and the most severe region in the collapse stage is the rear end of the downstream segment after the bubble cloud sheds off. The impact load of the cavitation jet is mainly affected by the stand-off distance of the nozzle from the impinged target, while the nozzle outlet geometry also has an effect on the impact performance. Optimizing the stand-off distance and the outlet geometry of the nozzles is found to be a good way to improve the performance of the cavitation jet.

\section{Introduction}

High-pressure jet technology has the characteristics of high energy density, strong impact force, and high controllability. It is widely used in petroleum engineering, ship cleaning, and metal material-strengthening engineering fields [1-3]. When the high-pressure jet is submerged, the velocity of the nozzle outlet can usually reach more than $200 \mathrm{~m} / \mathrm{s}$. At this time, a high-velocity gradient is formed between the highspeed jet at the nozzle outlet and the surrounding static fluid. Shearing layer is formed between the dynamic and the static region, which produces vortex rings with very low central pressure. Liquid phase transforms to vapor phase in the lowpressure region and cavitation happens [4]. Studies have shown that the collapse of cavitation is accompanied by extremely strong shock waves and microjets, which creates shock and vibration in hydraulic machines [5-8]. In the pumps with high specific speed, cavitation caused by the tip vortex is usually found when the pump works under low cavitation number conditions $[9,10]$. These phenomena can cause cavitation damage to the impact material $[11,12]$. On the other hand, when cavitation is controlled and used properly, it can drastically improve the efficiency of jet cutting, rust removal, and peening process [13-16]. 
At present, many scholars pay attention to the impact of high-pressure jets, aiming at optimizing high-pressure jet cutting and scouring performance. By optimizing the cavity of high-pressure nozzle for oil drilling, a method that makes use of self-excited oscillation of cavitation flow is proposed to increase the cutting efficiency of the jets. The efficiency for the rock breaking is increased by 1-2 times [17]. In the field of cavitation water jet peening (CWJP), the process parameters are optimized with reference to the fatigue strength, surface roughness, and residual stress depth of the material surface after peening [18]. The jet target distance and impact time were analyzed in detail. The effect of nozzle roughness on the erosion performance is also studied [19]. It was found that under the different inlet pressure conditions, the roughness of the inner wall of the nozzle corresponding to the strongest erosion performance was also different. Besides, cavitation jet is also used for the material test, which evaluates the strength and the anticavitation performance of the metals [20-23].

In order to improve the impact of high-pressure cavitation jets and to grasp the flow characteristics of cavitation jets as well as the mechanism of cavitation formation and collapse, many researchers have studied the velocity field, pressure distribution, and cavitation morphology of cavitation jets. Soyama et al. [24] considered that mastering the dynamic characteristics of high-pressure cavitation jets is of great significance for improving the performance of cavitation jets. They studied the cavitation morphology of highpressure cavitation jets produced by three different nozzles by high-speed photography. The pressure test paper is used to measure the distribution of the impact pressure generated by the collapse of the bubble downstream of the nozzle. It is found that the shape of the bubble generated in the jet is greatly affected by the nozzle structure, and there is a continuous bubble group at the exit of the nozzle, but it will break and shed off after a certain distance. The pressure distribution downstream of the nozzle has two peaks. It is found that this distribution characteristic may be related to the unstable shedding phenomenon of the bubble group.

A transparent shrink-type nozzle using acrylic resin is used to observe the internal cavitation of the high-pressure jet nozzle, and high-frequency and low-frequency signals are obtained from the image grayscale of the high-speed photography [25]. By comparison, it is found that the low-frequency signal is consistent with the pulsation of the plunger pump, and the high-frequency signal is related to the frequency of the cavity shedding. By monitoring the variation of cavity length over time, low-frequency signal corresponding to the pressure fluctuations of the plunger pump and the highfrequency signal related to the cavity shedding were also obtained [26]. Based on the high-speed photography technology, the phenomenon of cavitation shedding in the jet is further studied, and a function of shedding frequency corresponding to jet pressure and nozzle geometry is established, which is verified through experimental analysis [27]. At the same time, optical technology was used to capture the flashing phenomenon while the bubbles collapse, which proves the existence of the high-energy shock wave. Watanabe et al. [28] applied POD image processing technology to high-speed image analysis of cavitation jets and captured the location of cavitation collapse. Fujisawa et al. [13] conducted high-speed photography and schlieren shooting on the high-pressure submerged jet. The resulting bubble collapse position was similar to that obtained by Ryuta Watanabe, while the schlieren camera captured the shock wave generated by the collapse of the bubbles.

However, the research on the unsteady characteristics of high-pressure cavitation jets mainly proves the high-frequency shedding phenomenon of submerged high-pressure jet. The study on the relationship between the unsteady cavitation phenomenon and the nozzle divergent angle as well as the working condition is lacking. In addition, the research for the impact load characteristics of the submerged cavitation jet on the impinged surface under different operating conditions is lacking. In this paper, the unsteady characteristics of high-pressure cavitation jets under three different angles and different cavitation numbers are analyzed by means of highspeed photography and high-response pressure transducer detection. The frequency of cavitation shedding under different conditions is extracted and compared by analyzing the grayscale data of cavitation cloud, and the collapse impact of the cavitating bubbles is studied.

\section{Experimental Apparatus and Methods}

2.1. High-Pressure System for the Submerged Cavitation Jet. Figure 1 shows the cavitation jet experiment platform and test system. The system uses Italian AR high-pressure piston pump to provide high pressure to the jet. The maximum working pressure of the piston pump is $50 \mathrm{MPa}$, the rated rotating speed is $1450 \mathrm{rpm}$, and the flow rate is $0.00025 \mathrm{~m}^{3} / \mathrm{s}$. The water used in the experiment is pure water and the temperature is $25^{\circ} \mathrm{C}$. The impurities are removed by the Y-type filter before flowing into the plunger pump; the pressure relief valve and the pressure gauge are connected downstream. The upstream pressure of the nozzle is controlled by adjusting the speed of the plunger pump. A pressure gauge is set between the decompression valve and the nozzle, which displays the instantaneous upstream pressure of the nozzle. The working pressure is controlled by varying the rotating speed of the piston pump, which is realized by setting the motor speed using an inverter. When the experiment pressure is decided, the motor speed is increased gradually till the pressure on the gauge reached the objective value. Figure 2 shows the relationship between the rotational speed and the pump pressure when the jet is generated by different nozzles.

In order to avoid the influence of the upstream pipe bending on the nozzle pressure, a $300 \mathrm{~mm}$ stainless steel straight pipe is connected upstream of the nozzle. The jet platform is divided into two parts: a test water tank and a water storage tank. The water storage tank for supplying water to the plunger pump is located at the bottom of the test water tank. In order to facilitate the visual study of the submerged jet, the test tank is made of transparent material, polymethyl methacrylate, which has a refractive index close to that of water and can effectively avoid positional errors caused by photography. In order to ensure that the outlet can return to the same position after each nozzle change, the 


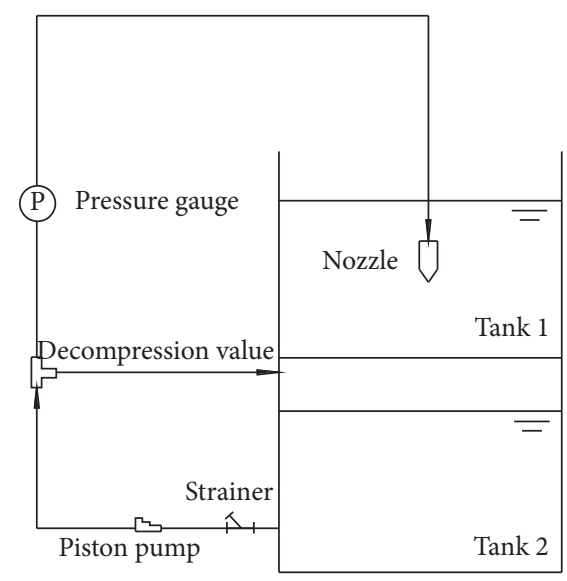

(a)

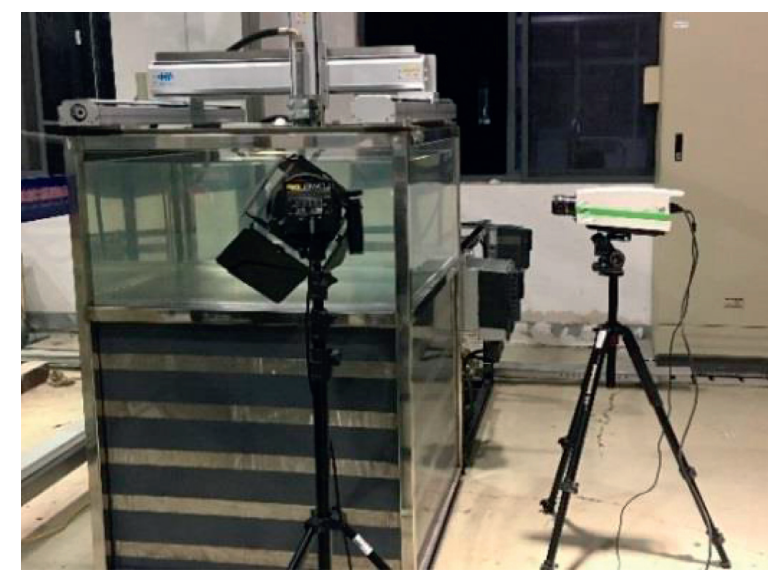

(b)

Figure 1: Test bench for high-pressure submerged cavitation jet. (a) Schematic diagram. (b) Photo of the experiment platform.

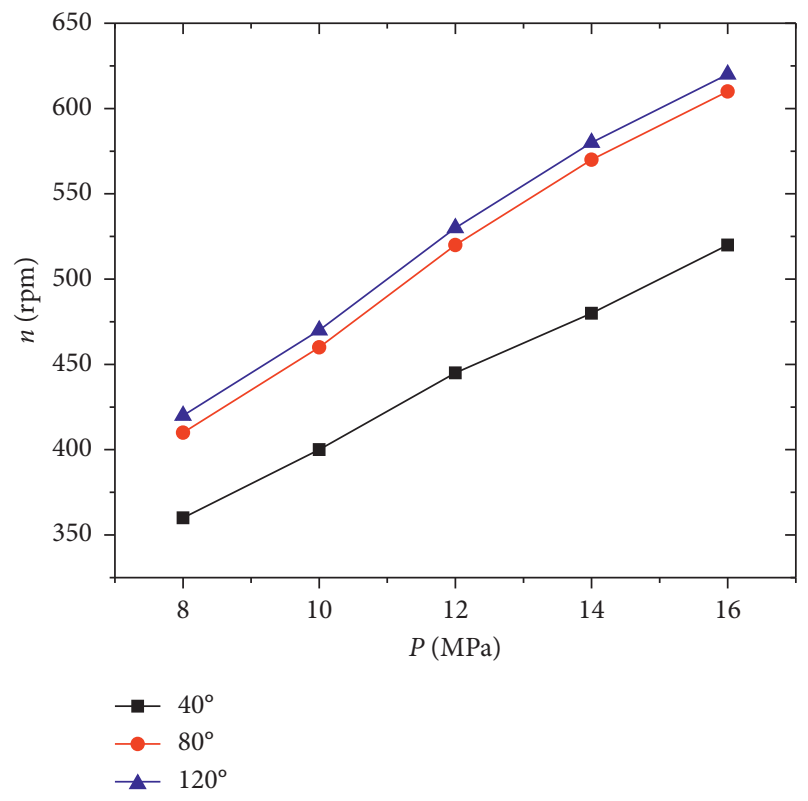

FIGURE 2: Relationship between pressure and rotating speed.

nozzle is fixed on the three-degree-of-freedom moving slide by the clamp, and the repeating positioning accuracy of the slide is $0.01-0.02 \mathrm{~mm}$. When the nozzle and the environment of the submerged jet are kept unchanged, the cavitation intensity is only correlated with the cavitation number which is defined as follows: $\sigma=\left(p_{\infty}-p_{v}\right) /\left(p_{n}-p_{\infty}\right)$, where $p_{\infty}$ is the pressure at the downstream of the nozzle outlet, $p_{v}$ is the saturated vapor pressure at the temperature of the jet, and $p_{n}$ is the pressure at the upstream of the nozzle. As the temperature and the downstream pressure are not changed during the experiment, the cavitation number is varied by changing the upstream pressure.

2.2. Process for High-Speed Photography. Figure 3 shows the convergent-divergent nozzle commonly used in cavitation jets. The key parameters include the throat diameter $d$, the

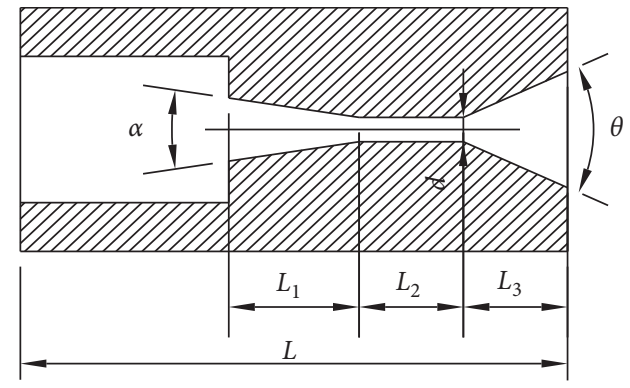

Figure 3: Schematic diagram of the nozzle structure.

convergent angle $\beta$, the divergent angle $\alpha$, and the corresponding length of the three stages. In this paper, the effects of divergence angle on cavitation jet are studied. Three different divergent angles $\alpha=40^{\circ}, 80^{\circ}$, and $120^{\circ}$ are studied. The other parameters are selected as follows: $D=6 \mathrm{~mm}$, $d=1 \mathrm{~mm}, \beta=13.5^{\circ}, L_{1}=5 \mathrm{~mm}, L_{2}=4 \mathrm{~mm}$, and $L_{3}=4 \mathrm{~mm}$. High-speed photography is conducted using a OLYMPUS high-speed camera. It is illuminated by a $150 \mathrm{~W}$ LED spotlight, which irradiates from the back side of the tank. The transparency of the interface between the bubble and the water is relatively low, and the reflection for the light is strong. As a result, the cavitation region in the photo is relatively bright. The shooting area is $272 \times 352$ pix, the shooting frequency is $20000 \mathrm{fps}$, and the exposure time is $50 \mu \mathrm{sec}$. The image recording is started after the motor reaches the stable rotating speed, and the recording time of each group of data is more than 1 second. In high-frequency shooting, the interval between adjacent frames is extremely short, which is convenient for recording the process of bubble cavitation generation, shedding off, and collapse. In order to quantify the spatial characteristics of the reaction bubble development, the photographs were calibrated in the experiment, as shown in Figure 4. The scale ruler is located in the axis plane of the nozzle, and the direction is perpendicular to the axis of the camera lens. The positions of the lens and the nozzle are kept unchanged during the image recording. After the data is captured, the length of $60 \mathrm{~mm}$ is selected in the postprocessing software i-SPEED, and the 


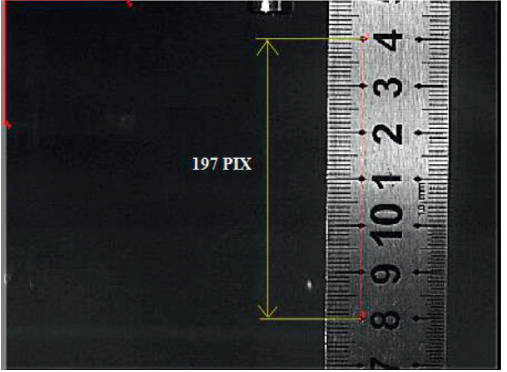

FIgURE 4: Image size calibration.

number of pixels spanned is 197 pix. From this, it is known that a single pixel occupies a square with dimension of $0.305 \mathrm{~mm} \times 0.305 \mathrm{~mm}$.

2.3. Detection for Shock Impact of the Cavitation Jet. The impact loads caused by the shock during the collapse of the bubbles are measured using a high-response pressure transducer PCB 102B03, which is flush-mounted on the surface of stainless steel plate. The pressure transducer has a rise time of $1 \mu \mathrm{s}$, a sensitivity of $0.07 \mathrm{mV} / \mathrm{kPa}$, a resonant frequency of $500 \mathrm{kHz}$, and a diameter for sensitive area of $5 \mathrm{~mm}$. The sensor is powered by a constant direct current of $4 \mathrm{~mA}$ provided by using a power supply/signal conditioner produced by LANCE, and the output voltage signal is collected using a data acquisition card HSJ2010 with a sampling rate of $250 \mathrm{kHz}$. The pressure signals of different nozzles with different cavitation number and stand-off distances are recorded and analyzed to avoid the damage of the sensor by the cavitation impact; the probe is set as $1 \mathrm{~mm}$ recessed relative to the impinged plane. On the other hand, the experimental procedure is optimized to limit the exposure time of the sensor to the cavitation jet. To make sure that the accuracy of the sensor is not affected by the cavitation impact, one set of data with the same working condition is collected before and after all the experiments.

\section{Results and Analysis}

3.1. Cavitation Cloud Distribution of the Submerged Jet under Different Pressures. Figure 5 shows the dynamic process of a bubble cloud generated by a nozzle with a divergent angle of $40^{\circ}$ under four different cavitation numbers. The interval between adjacent photos is $50 \mu \mathrm{s}$, and the total time span is $0.9 \mathrm{~ms}$, which includes the complete shedding-off period. It can be seen that the distribution of the bubble cloud under different pressures has a large difference, mainly in the length and diameter of the bubble cluster, and there is also a certain difference in the location of the cavity. In general, as the pressure increases, the cavitation intensity increases gradually, and the size of the cavitation cloud gradually increases. The position where the shedding occurs slightly moves downstream, and the shedding period is slightly extended. It can be seen from Figure 5(a) that the initial cavitation cloud $(t=0 \mu \mathrm{s})$ is basically continuous, and there is a slender water column near the nozzle exit, which is the jet core region with the highest velocity. Due to the viscosity of the water, a vortex ring is generated at the exit of the nozzle $(Y=0$ to $10 \mathrm{~mm})$ under high-speed shear which spreads to the surroundings. Since the scale of the vortex ring is as small as the diameter of the nozzle throat and the frequency of the vortex generation is high, it is difficult to identify it in the high-speed photos. A better way to reveal the development of the Rankin vortex is numerical simulation. According to the characteristics of the jet, the vortex pairing happens while the Rankin vortex moves downstream. This phenomenon makes it possible to visit the merged vortex which has a much larger scale than the original Rankin vortex. Under the influence of the lowpressure zone in the vortex core center, the water here changes to vapor, and the cavitation cloud diffuses with the vortex ring under the effect of turbulent flow. The columnar bubble cloud starts to expand from the position of $Y=10 \mathrm{~mm}$; the maximum is reached at the position of $Y=20 \mathrm{~mm}$ and then it begins to shrink. The top of the bubble cloud is tapered because the velocity at the center is the highest and the bubble cloud moves the fastest under the drag of the liquid. When it develops to the time of $t=50 \mu \mathrm{s}$, the bubble cloud breaks at the position of the nozzle exit section $Y=5-10 \mathrm{~mm}$, and the two sections continue to develop after the fracture. At the front end, the detached cavitation cloud gradually collapses as the pressure rises during the movement toward downstream. At the same time, it diffuses under the effect of turbulence, and the edge contour of the cavitation cloud becomes blurred. Under the combined action of diffusion and collapse, the area of the bubble cloud in the shedding segment decreased rapidly. At $400 \mu$ s, only the annular vapor cloud is remained in the relatively low-pressure region. The bubble at the exit section of the nozzle rapidly grows after the fracture and extends downstream. It has a columnar structure before the time of $150 \mu$ s and then expands and spreads in the latter part. As shown in Figure 5(b), when the cavitation number is reduced to $\sigma=0.0099$, the bubble cloud shedding-off position moves slightly downstream, and the breakage occurs at $Y=10-15 \mathrm{~mm}$. The development trend of the bubble near the nozzle segment after fracture is similar to that at $\sigma=0.0124$. The collapse of the upper end of the shedding section is intensified, so that the spacing between the two sections of the bubble cloud gradually increases, indicating that the pressure fluctuates at $Y=30 \mathrm{~mm}$ to accelerate the collapse of the bubble. The next shedding occurs at $800 \mu \mathrm{s}$, and the shaded bubbles have not disappeared when the cloud moves to the position $Y=52 \mathrm{~mm}$. Compared with $\sigma=0.0124$, the length of the bubble cloud increased and the development cycle was slightly extended. Under the condition of $\sigma=0.0082$, due to the increase of flow coefficient and flow rate, the cavitation phenomenon is rapidly intensified, while the diameter and length of the cavitation become significantly larger than the above two pressure conditions. In the case of $\sigma=0.0070$, the jet velocity is faster, and the bubble generation is more intense, so that the bubble can move to a position further downstream before collapse.

Convergent-divergent nozzles are widely used in cavitation jets. Compared to other types of nozzles, the cavitation performance of this type of nozzle is higher under the 


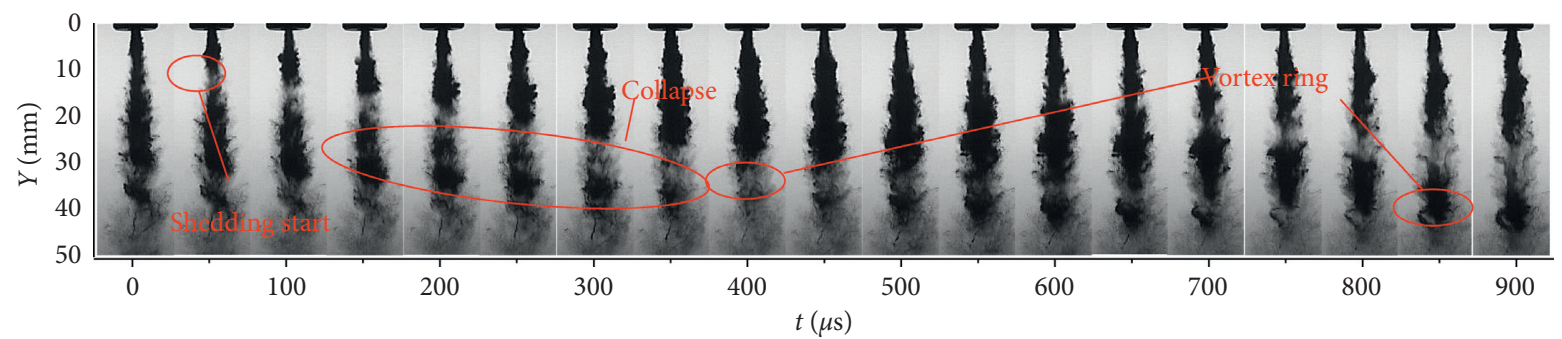

(a)

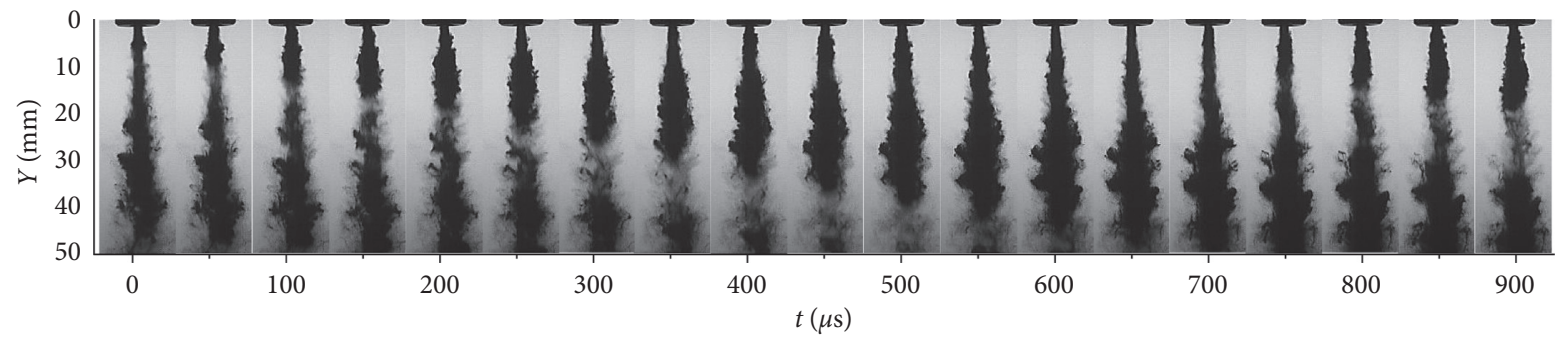

(b)

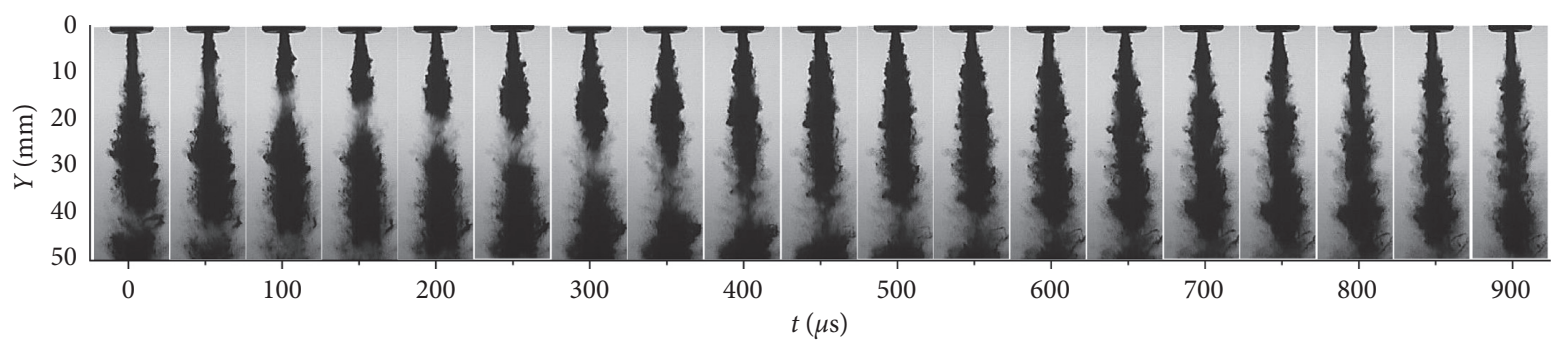

(c)

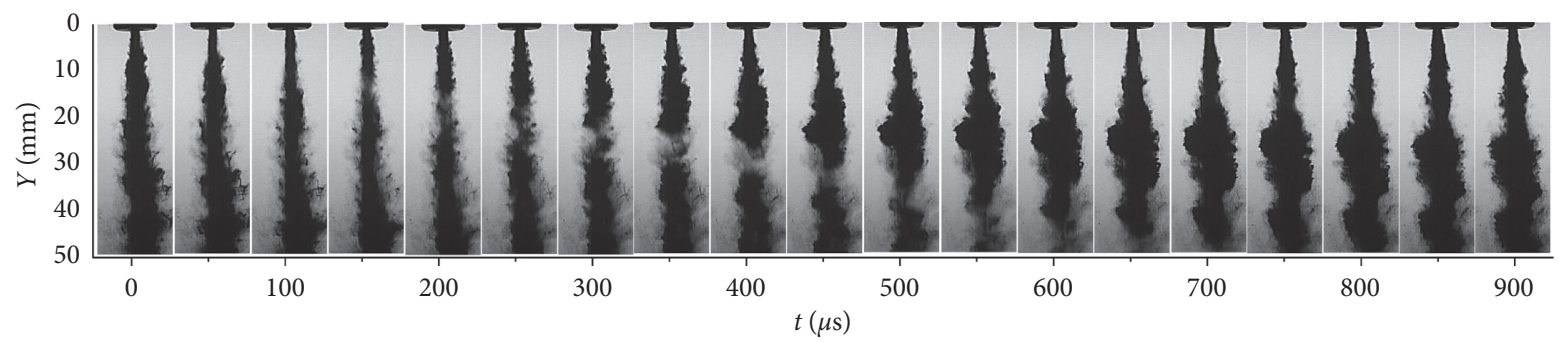

(d)

Figure 5: Cavitation cloud distribution of nozzle with divergent angle of $40^{\circ}$ under different pressures. (a) $\sigma=0.0124$, (b) $\sigma=0.0099$, (c) $\sigma=0.0082$, and (d) $\sigma=0.0070$.

same power conditions. In this paper, high-speed photography is conducted to study the influence of the exit divergent angle of the nozzle on the jet cavitation cloud. Figure 6 shows a transient photograph of a bubble cloud generated by a submerged jet using nozzle with divergent angle of $40^{\circ}, 80^{\circ}$, and $120^{\circ}$. The cavitation number is kept as $\sigma=0.0062$, and the interval between the two adjacent photographs is $50 \mu \mathrm{s}$. As shown in Figure 6(a), at the initial time $(t=0 \mu \mathrm{s})$, the bubble cloud is a columnar structure whose diameter gradually increases from the vicinity of the nozzle to the downstream. Then, shedding began to occur near the position $Y=20 \mathrm{~mm}$. During the shedding process, the rear end of the bubble group in the downstream section collapsed rapidly, and the cloud structure of the bubble was bent, indicating that the turbulence at this position was severe and the pressure was relatively high. Due to the collapse of the tail of the shedding section, the distance between the separated bubble clouds gradually increases, and the upstream bubble cloud collapses and shrinks meanwhile. Comparing the cavity of this nozzle to the others, it can be found that the diameter of the cavity is the largest in case of the nozzle with an angle of $40^{\circ}$, indicating that the diffusion of the bubbles in the jet is faster than the other two nozzles. The shape of the cavity of $80^{\circ}$ nozzle is close to that of the $40^{\circ}$ nozzle, but the contour of the bubble is clearer, indicating that the distribution of the bubble is more concentrated, less affected by the surrounding eddies and turbulence, and the diffusion is slower. For the cavitation jet of the $80^{\circ}$ nozzle, most of the bubbles collapse before reaching the position of $Y=60 \mathrm{~mm}$, indicating that this 


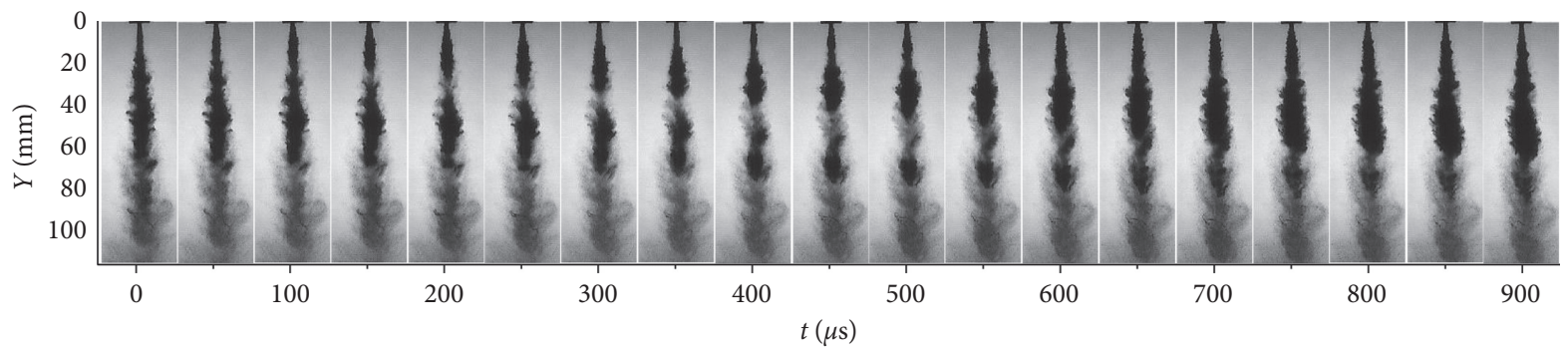

(a)

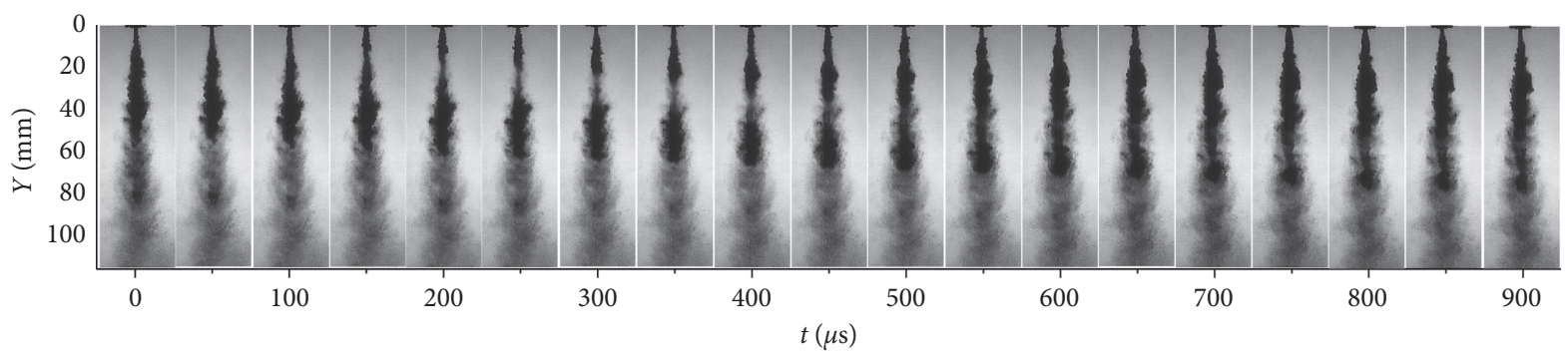

(b)

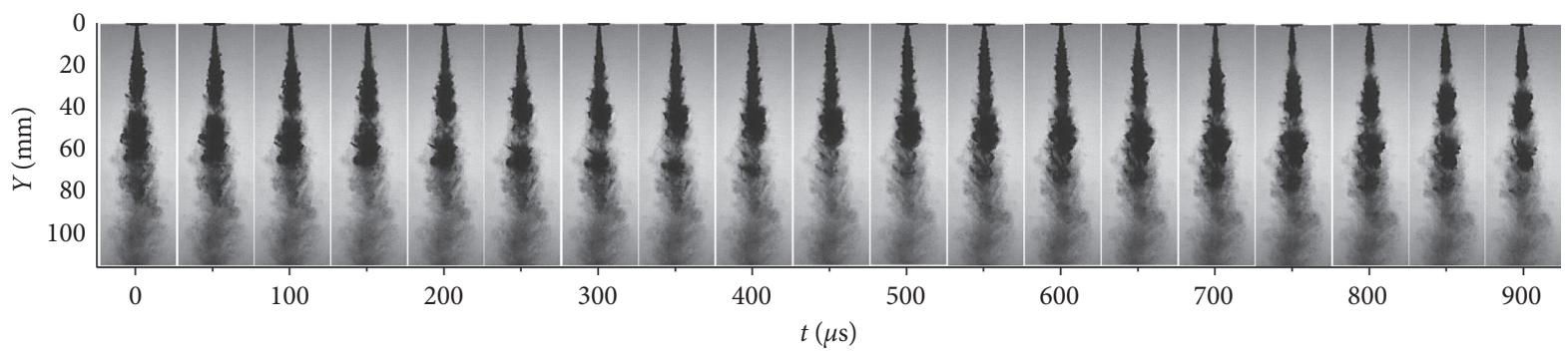

(c)

Figure 6: Cavitation cloud distribution of three different nozzles $(\sigma=0.0062)$. (a) Divergent angle $\alpha=40^{\circ}$. (b) Divergent angle $\alpha=80^{\circ}$. (c) Divergent angle $\alpha=120^{\circ}$.

position is the optimal target distance for cutting or peening using cavitation jet by this nozzle. Compared with the first two nozzles, the bubble shape of the nozzle with a divergence angle of $120^{\circ}$ is quite different. After the shedding, the cavity spreads out and collapses slowly, and the next shedding starts before the collapse of the former separated cavitation cloud. The diameter of the cavity is smaller while the periodical character is weaker. In summary, the angle of expansion of the angle nozzle has a significant effect on nozzle performance, especially on cavitation performance. The smaller the divergent angle, the better the cavitation performance, but it also causes the instability of the flow, and the continuity of the bubble is poor. When the divergent angle is too large, the restraining force of the outlet section on the shear layer is insufficient, which will cause decline of the cavitation performance.

3.2. Grayscale Analysis on the Image of the Submerged Cavitation Jet. In order to extract the spatial distribution change of the bubble in a very short time, the photo obtained by high-speed photography is converted into a gray matrix by MATLAB code. Since the light transmittance of the bubble is much lower than that of the liquid water, when the light of the halogen lamp is projected to the vapor-liquid interface of the bubbles, the vapor bubble reflects part of the transmitted light to the camera lens, and the higher the cavitation concentration, the higher the imaging brightness that can be obtained by the camera. The grayscale processed image corresponds to one gray value per pixel, the gray value is positively correlated with the brightness in the photo, and the higher the bubble density is, the higher the gray value is. On this basis, the grayscale intensity difference between the two photos at the adjacent time can be used to reflect the growth and disappearance of the bubble. In this paper, FDM analysis is carried out on the low pump pressure $(\sigma=0.0124)$ of $40^{\circ}$ divergent angle nozzle. As shown in Figures 7 and 8, the development process corresponds to the first $250 \mu$ s period of Figure 5(a). The scale value in the figure is proportional to the growth rate of the bubble; the positive value indicates the growth of the bubble, and the negative value indicates the collapse of the bubble. A more pronounced annular growth zone can be observed near the nozzle, which is the cavitation generated by the shear between the high-speed fluid and the stationary fluid. The surrounding pressure of the shear layer is quickly replaced by the environmental pressure, so the growth zone shows a certain change in strength and weakness with time. At the 

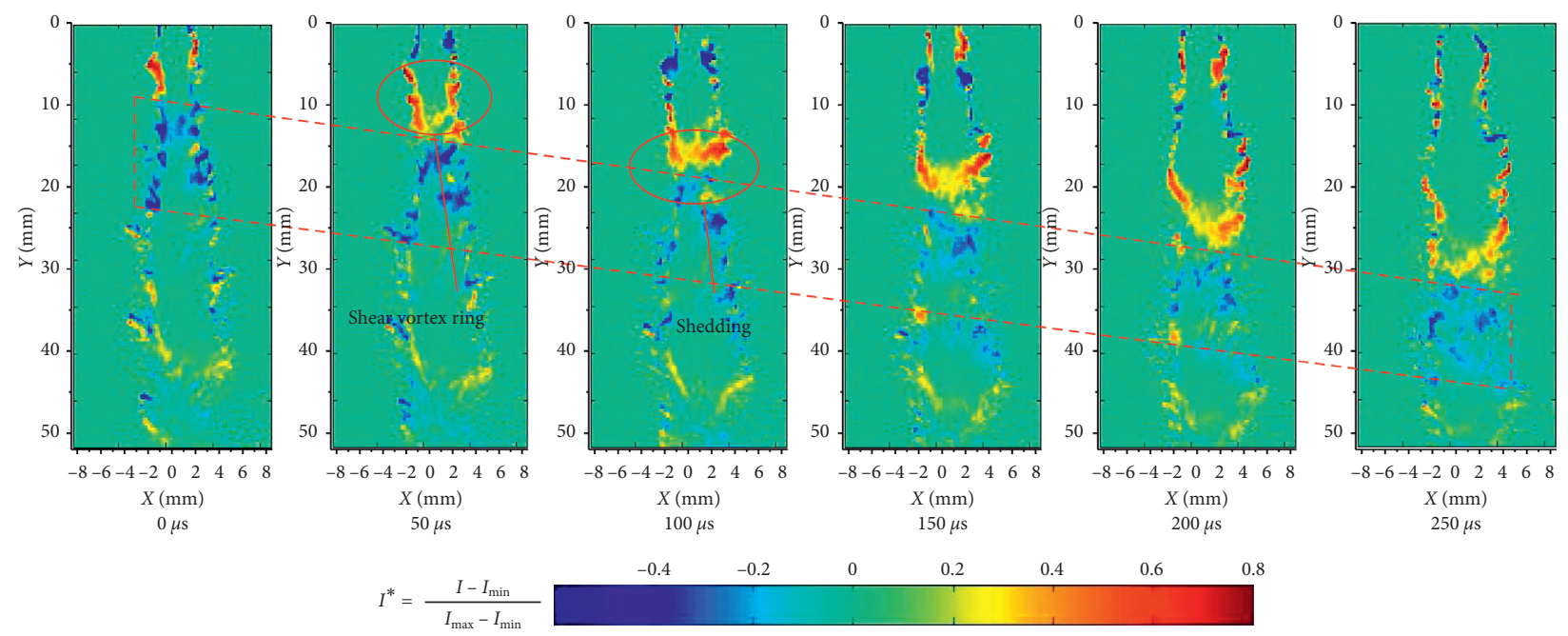

FIGURE 7: Image treated with the frame difference method.

time of $50 \mu \mathrm{s}$ in Figure 7, there is a relatively obvious grayscale reduction near the nozzle exit, which is due to the sudden expansion of the bubble cloud in the exit section of the nozzle, so that the bubble cloud has a local short-term separation. The blue area indicated by the dotted line in the figure indicates the main area of the bubble collapse. As can be seen from Figure 5(a), the collapse of the bubble mainly occurs after the bubble cloud is broken, and the position is concentrated on the rear end of the shed cavitation cloud. This is consistent with the research conclusion of Fujisawa et al. They recorded the shock wave generated by the collapse of the high-pressure submerged cavitation jet by the schlieren. The shock wave is mainly located at the rear end of the shed bubble cloud.

In order to qualitatively compare the influence of various parameters on cavitation, this paper presents the extraction of the gray matrix of 2000 cavitation cloud photos with time interval of $50 \mu \mathrm{s}$, and the time averaged value is calculated. The dimensionless processing of the gray value I is performed to obtain the grayscale intensity value $I^{*}=\left(I-I_{\min }\right) /\left(I_{\max }-I_{\min }\right)$. Through the grayscale intensity map, the distribution of the cavitation bubbles under each pressure can be more clearly observed. The higher the grayscale value is, the more severe the cavitation occurs at the corresponding position during the analyzed period. Figure 8(a) shows the time-averaged grayscale distribution of the bubble image during the sampling period under three different pressures. It can be seen that as the pressure increases, the length and width of the bubble gradually increase, but the growth rate is not linear. It can be found from Figure 8(b) that the bubble concentration under each pressure is continuously increased from the nozzle outlet to the downstream and then gradually reduced with the effects of collapse and diffusion after reaching the peak value. In the case of $\sigma=0.0070$, the concentration of cavitation vapor reaches the peak first at $Y=20 \mathrm{~mm}$; the corresponding grayscale intensity value is 0.875 . When the cavitation number is $\sigma=0.0055$, the bubble concentration reaches the peak at position of $Y=20 \mathrm{~mm}-40 \mathrm{~mm}$; the maximum grayscale intensity value is around 0.9 . In the case of $\sigma=0.0049$, the bubble concentration is slightly higher than that of $\sigma=$ 0.0055

Figure 9 shows the time-averaged distribution of the cavitation cloud generated by three different nozzles under high pump pressure $(\sigma=0.0062)$. It is found by comparison that the 80-degree divergent angle nozzle has the widest range of coverage and relatively high intensity. For the cavitation jet of the nozzle with a divergent angle of $40^{\circ}$, the bubble cloud is more concentrated, while the cavity of the nozzle with a divergent angle of $120^{\circ}$ is more dispersed. It can be seen from Figure 9(b) that the difference in the bubble concentration distribution of the centerlines of the three nozzles is obvious. The cavitation bubble concentration of the nozzle with $120^{\circ}$ divergent angle is the lowest, while the $40^{\circ}$ nozzle shows the best cavitation performance.

\subsection{Analysis of the Shedding Frequency of Jet Cavitation} Cloud. As mentioned above, shedding-off phenomenon inevitably occurs in the submerged high-pressure cavitation jet flow. The growth, shedding, and collapse process of the bubble cavity have a certain period, and grasping the bubble shedding characteristic is helpful to improve the jet performance. At present, the parameters such as pump pressure, flow rate, and nozzle structure have been confirmed to have a great influence on the evolution period of the bubble. In this paper, the average value of the gray level for 2000 images is extracted by the image gray processing method, as shown in Figure 10. According to the spectrogram, the main frequency of the grayscale average is $1040 \mathrm{~Hz}$, indicating that the overall period of bubble growth, shedding, and collapse is $1040 \mathrm{~Hz}$. This is in line with the experimental results of Soyama et al. [24]. In their research, the development frequency of the submerged high-pressure jet was found as $0.5-2 \mathrm{kHz}$ by monitoring the length of the cavity. 

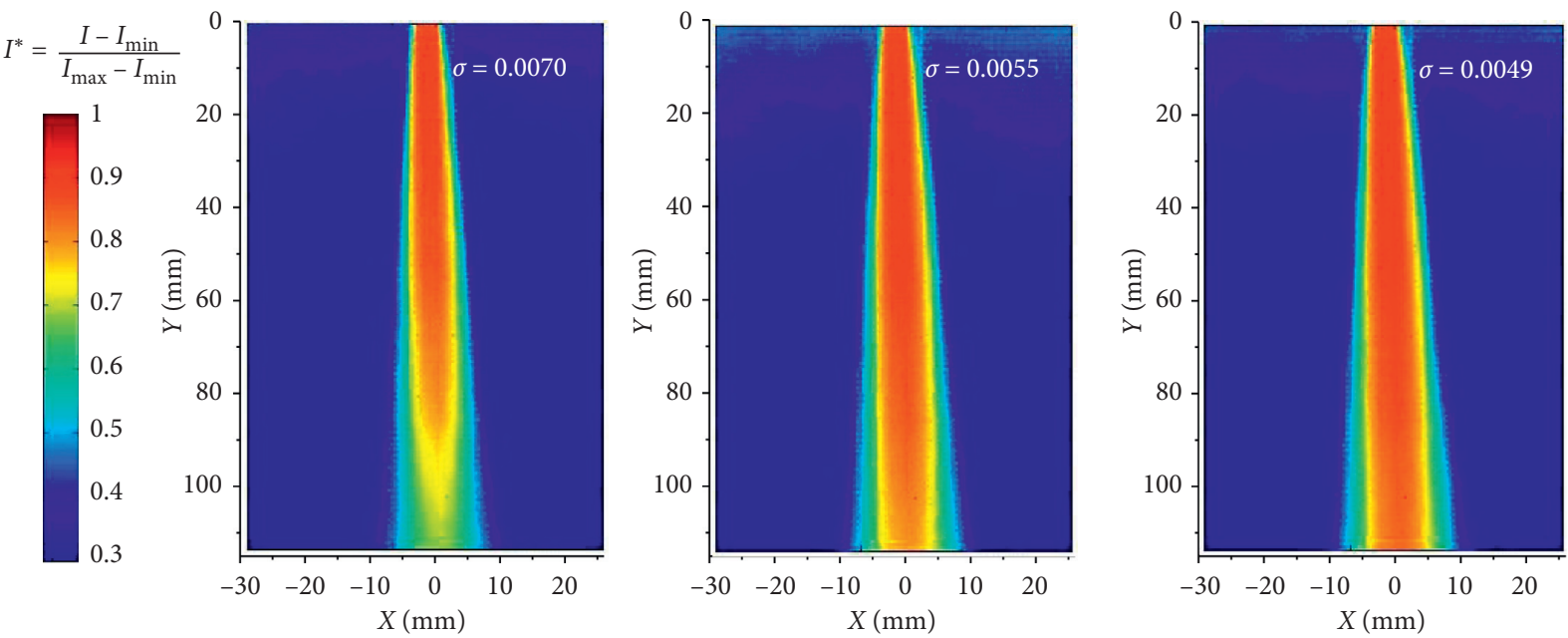

(a)

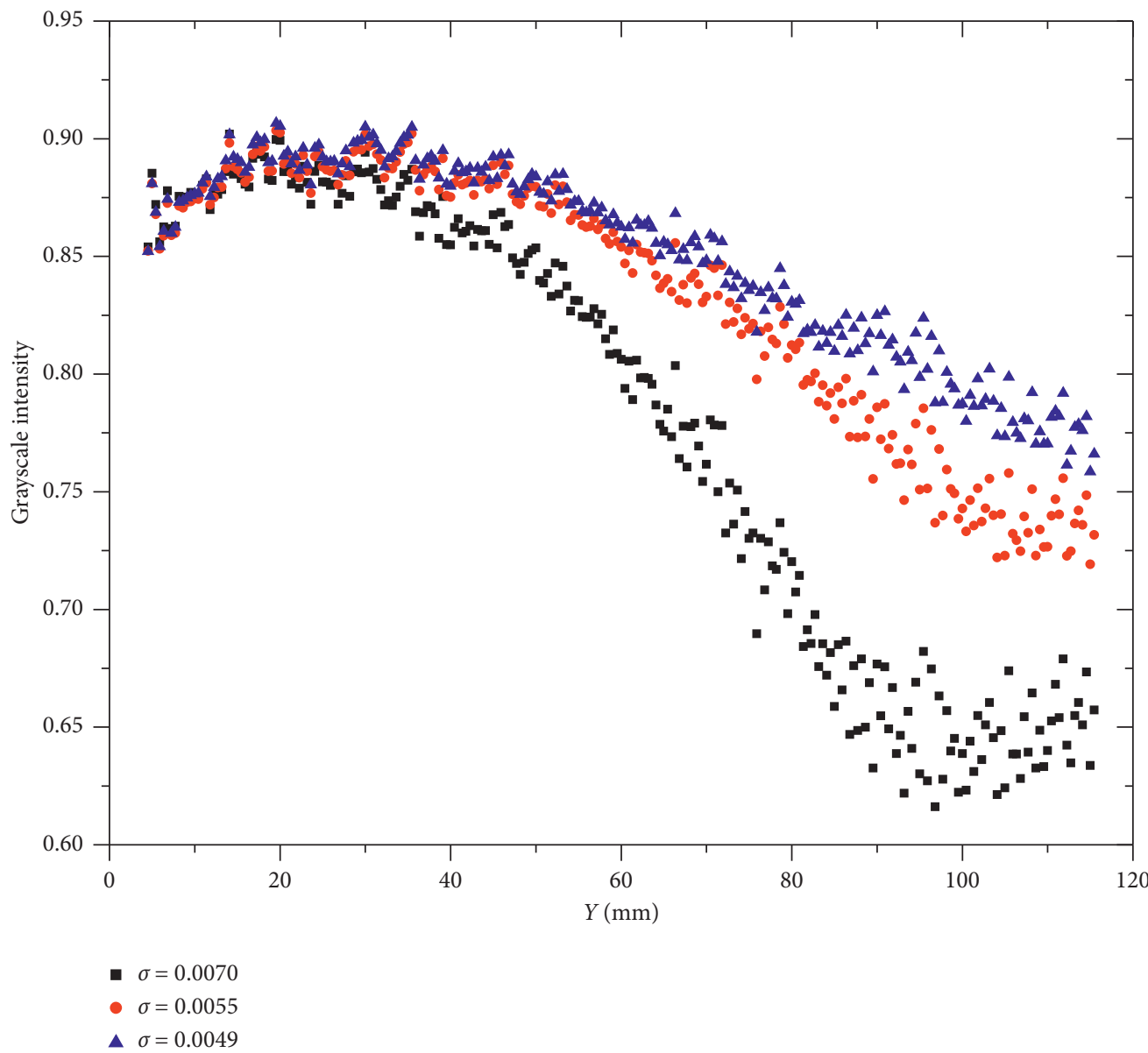

(b)

FiguRE 8: Time-averaged grayscale distribution of cavitation image with different jet pressure ( $40^{\circ}$ nozzle). (a) Contour of time-averaged grayscale. (b) Distribution of gray values on the central axis.

Figure 11 shows the spectrum of the bubble image of the three different nozzles under different pressures. It can be seen that in different cases, there is an obvious main frequency in the frequency spectrum obtained by FFT transformation, and the peak of the spectrum is mainly distributed between 0.5 and $2 \mathrm{kHz}$. With the increase of 

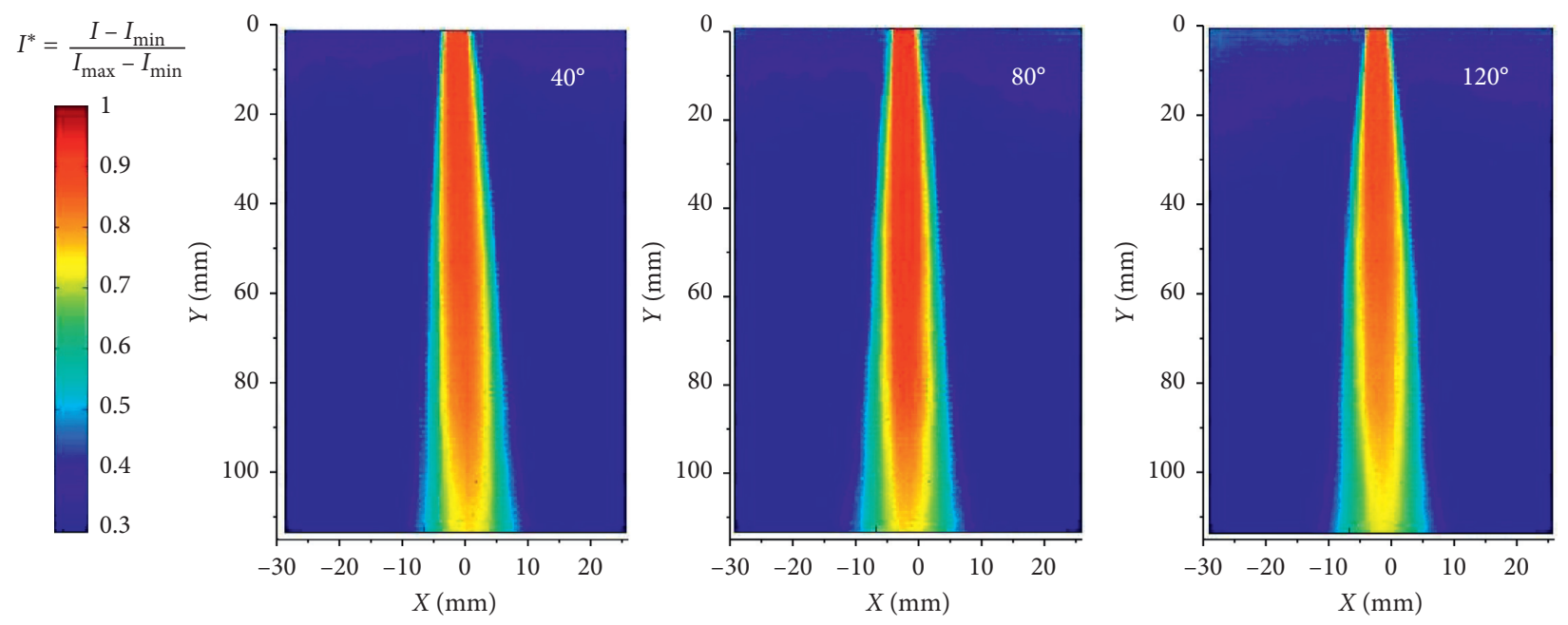

(a)

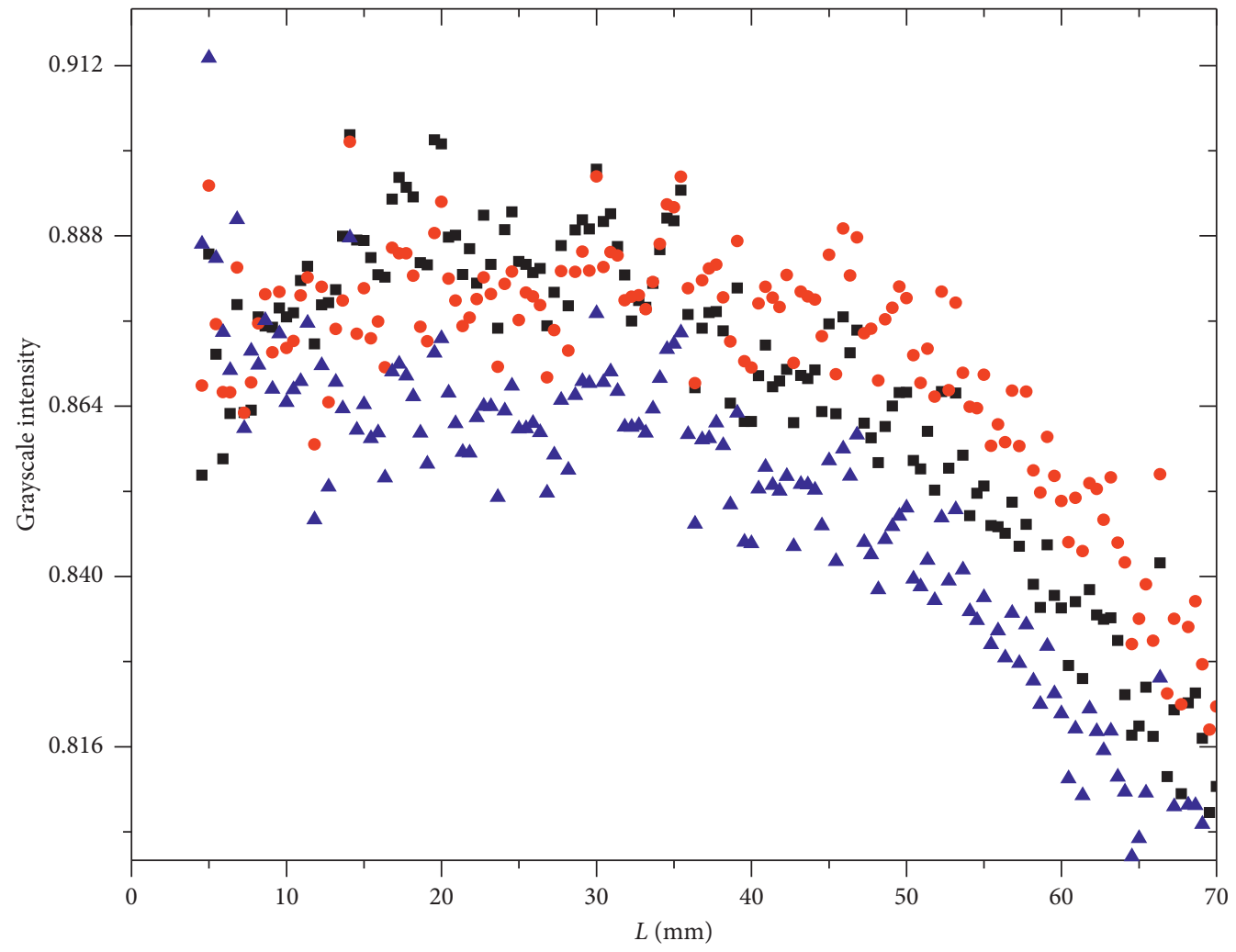

- $40^{\circ}$

- $80^{\circ}$

\ $120^{\circ}$

(b)

Figure 9: Time-averaged grayscale distribution of the cavitation image with different nozzles $(\sigma=0.0062)$. (a) Contour of time-averaged grayscale. (b) Distribution of grayscale values on the central axis.

pump pressure, the development period of the bubble generated by each nozzle is prolonged, and the frequency is obviously reduced. With the increase of the nozzle outlet divergent angle, the development period of the bubble is slightly shortened and the frequency is slightly increased. Hutli [27] proposed an empirical formula for the 


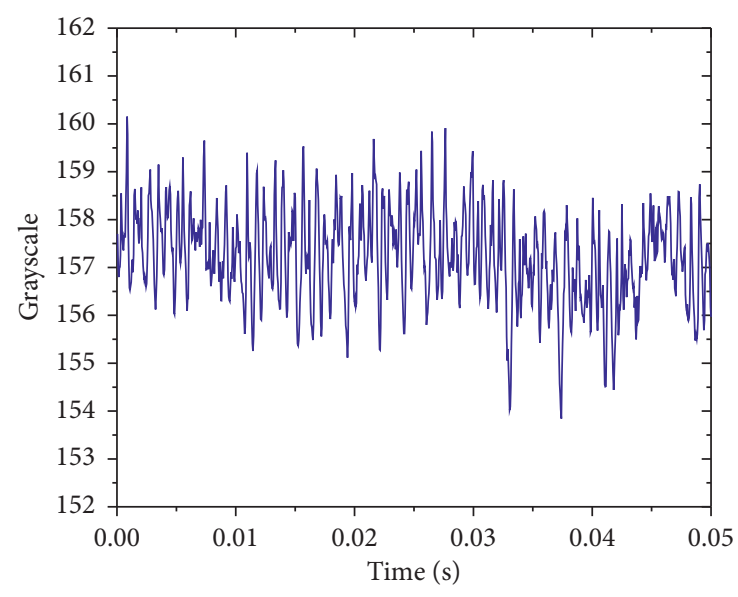

(a)

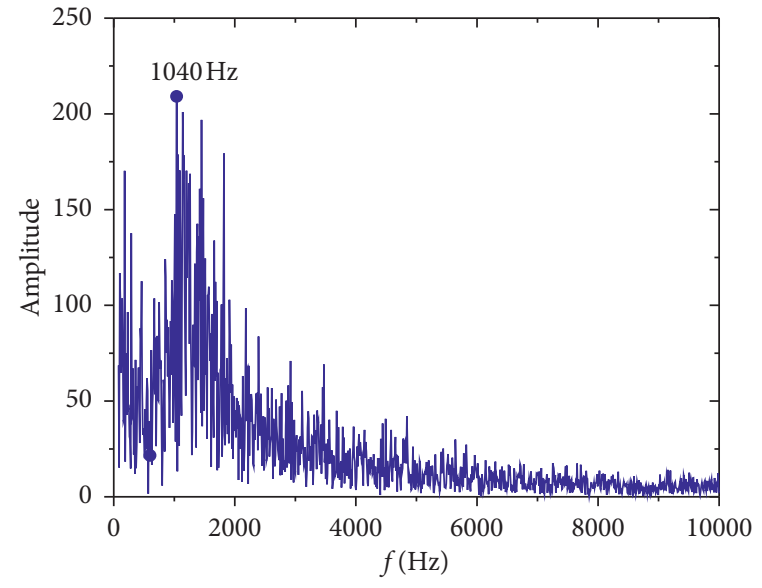

(b)

Figure 10: Global averaged grayscale value. (a) Time domain. (b) Frequency domain.
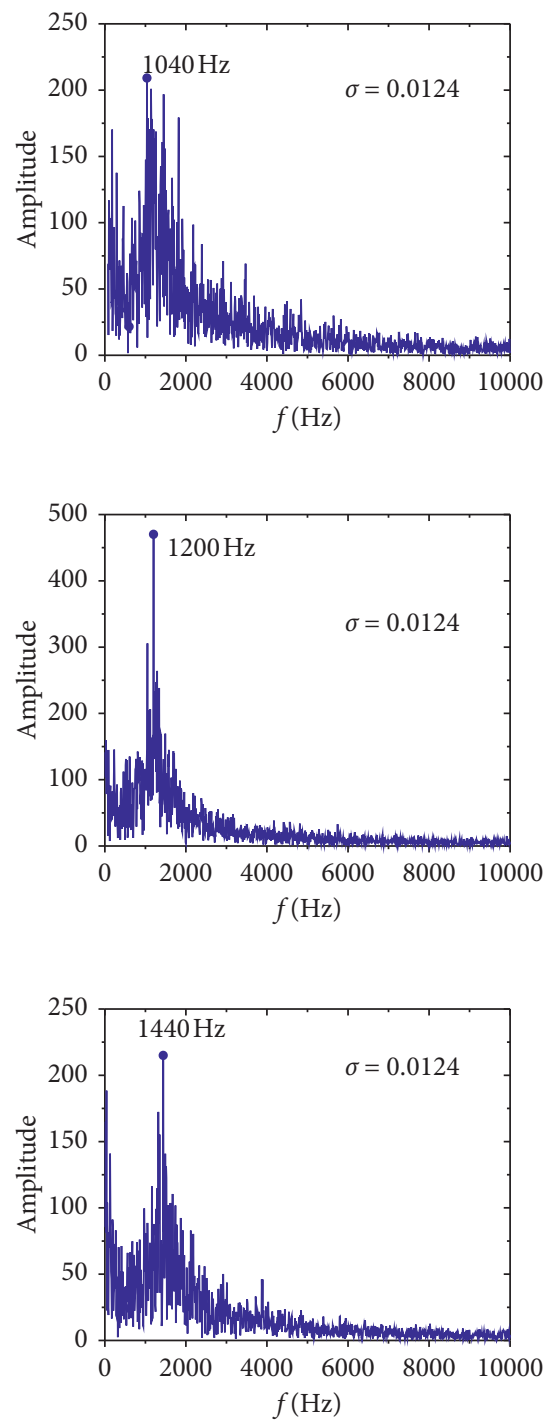

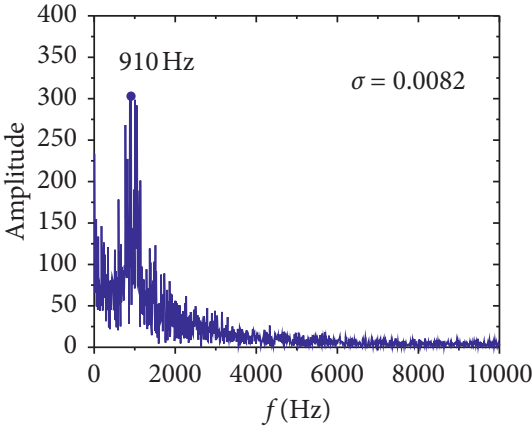

(a)
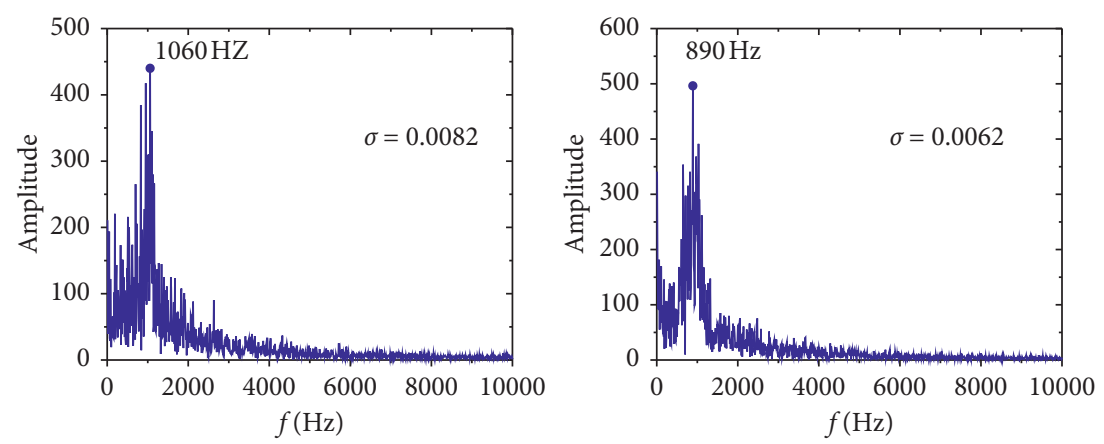

(b)
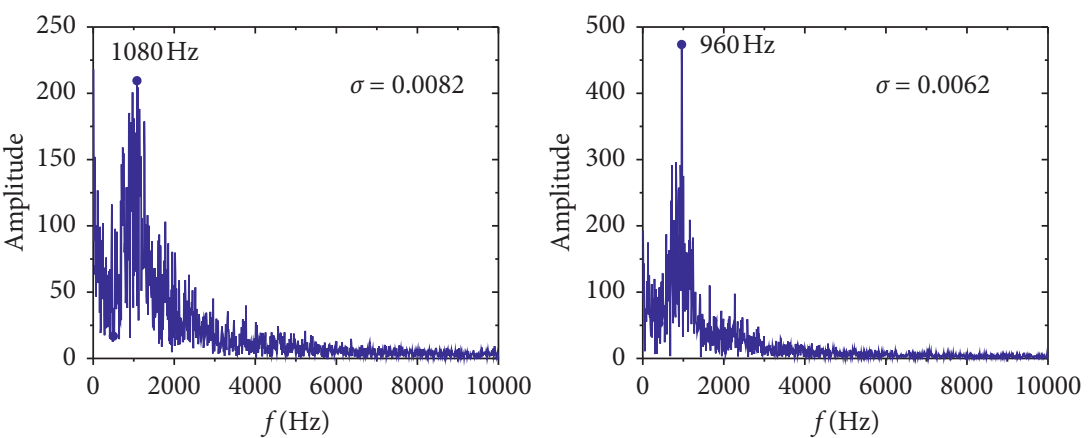

(c)

FIGURE 11: Frequency-domain diagram of global average grayscale with each nozzle at different pressures. (a) Nozzle with a divergent angle of $40^{\circ}$. (b) Nozzle with a divergent angle of $80^{\circ}$. (c) Nozzle with a divergent angle of $120^{\circ}$. 


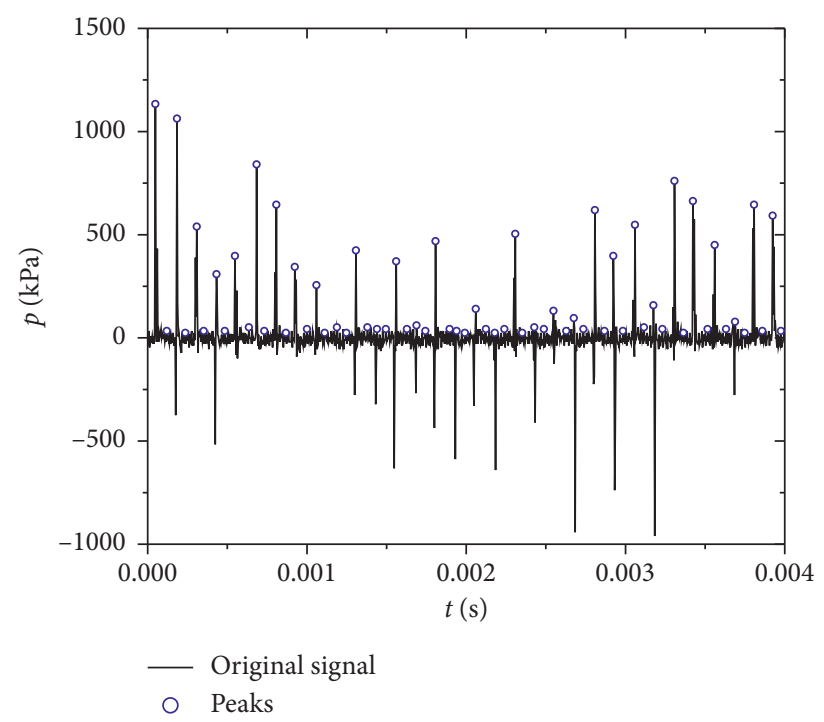

FIGURE 12: Distribution of the original pressure signal and the peaks.

relationship between the transient period of the submerged cavitation jet and the pump pressure: $t \mathrm{p}=1 / f=k \sqrt{P_{1}}$, where $P_{1}$ is the upstream pump pressure and $k$ is the coefficient determined by the nozzle geometry. Substituting the statistical results of this paper into the formula, it is found that when the geometric coefficient $k=0.0015$, the experimental value is consistent with the empirical calculated value, but there is still some error. Through the analysis, it is found that the pump pressure has a great influence on the development cycle of the cavitation jet, and also the nozzle exit angle has a little influence on the developing period.

3.4. Impact Load of the Cavitation Jet. Since the cavitation jet is usually applied for its high impact performance, the load strength of the shock caused by the bubble collapse is concerned by researchers. Researches for the erosion test and cavitation peening have shown that the impact performance can be affected by different factors, e.g., the cavitation number, stand-off distance from the target, and the geometry of nozzles. In this research, impact performances of cavitation jet generated with the abovementioned three nozzles are investigated. Pressure shocks under four different stand-off distance and two different cavitation numbers are recorded by the data collection system. Figure 12 shows the signal of a raw signal of the cavitation jet impact as well as the extracted peaks which represents the shock loads of the cavitation bubbles. It can be found from the figure that the amplitude of the peaks varies from zero to around $1 \mathrm{MPa}$, because the sensitive surface of the sensor is set as $1 \mathrm{~mm}$ recessed related to the impinged surface so that the sock wall cannot destroy the sensor. In fact, the pressure detected by the pressure transducer is an area-averaged value for the sensitive area which has a diameter of $5 \mathrm{~mm}$, while the minimum diameter of the bubble with a maximum diameter of $1 \mathrm{~mm}$ during collapse can be smaller than $200 \mu \mathrm{m}$. It infers that the pressure at the point where the bubble collapses can reach more than $600 \mathrm{MPa}$, which is higher than the fatigue stress of most of the aluminum alloys. From the pressure distribution, it can be found that some negative peaks always follow the positive peaks, which is caused by the ringing of the transducer. Jean-Pierre Franc et al. [29] had also encountered this problem and solved it by setting a locker while countering the peaks of the pressure. In the current research, the sampling frequency of the data collector is set as $250 \mathrm{kHz}$; the ringing has little effect on the statistics of the peaks and the locker is not used while countering the impact peaks.

Figures 13 and 14 show the number density of the pressure value versus the peak heights under different operating conditions, where 500000 (2s) of recorded data points are analyzed. Comparing the distributions for various stand-off distributions, nozzle geometries, and cavitation numbers, it can be found that generally, the stand-off distance has a great effect on the impact distribution of the cavitation jet on the target for the highly concentrated region of the density curves; the nozzle geometry affects the distribution mainly for the intensive impact region. The number density curve has a shape of Gaussian distribution, where the peaks are located between $600 \mathrm{kPa}$ and $1200 \mathrm{kPa}$ for all the cases analyzed in this research. When the cavitation number is dropped from 0.0124 to 0.0099 , the peak value and the impact frequency are increased slightly and the tendency for the impact peak number density distribution is not changed very much. During the experiment, the center point of the sensor is shifted $10 \mathrm{~mm}$ from the axis of the jet to protect the sensor from being overloaded. When the divergent angle of the nozzle is small $\left(40^{\circ}\right)$, the peak of the number density increases when the stand-off distance increase from $45 \mathrm{~mm}$ to $90 \mathrm{~mm}$. This is because that the region for the cavitation bubbles to collapse is 


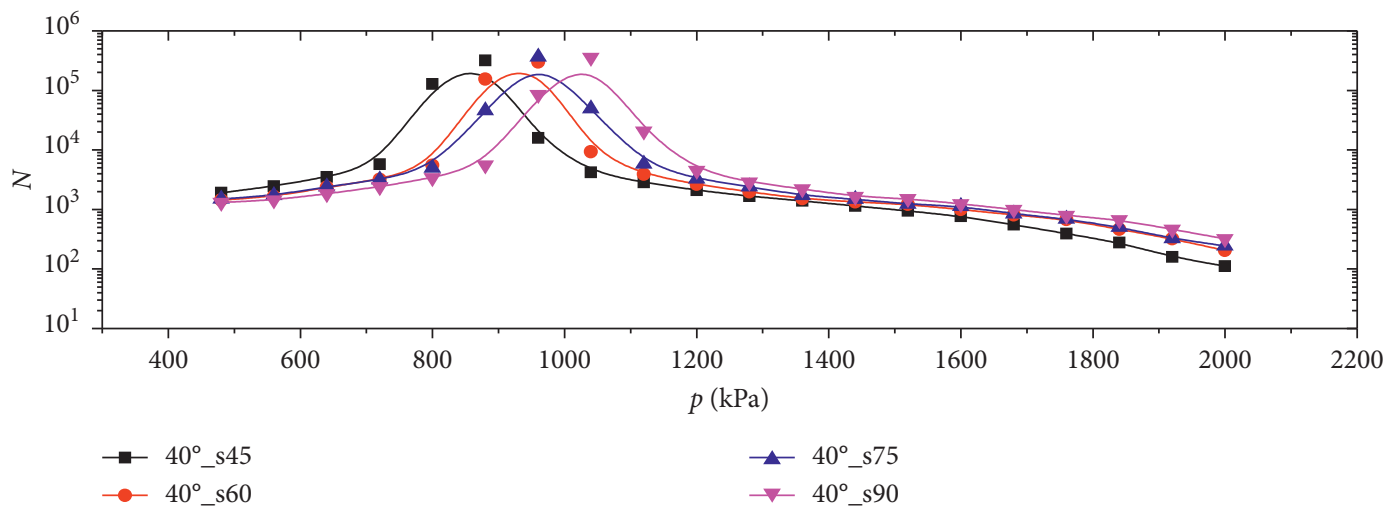

(a)

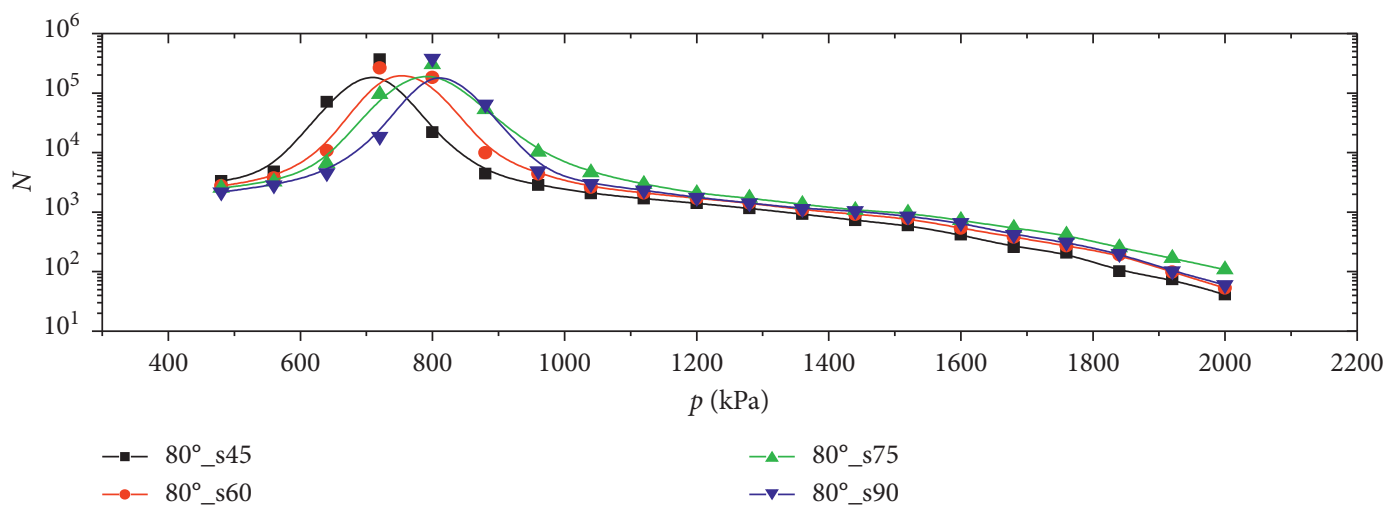

(b)

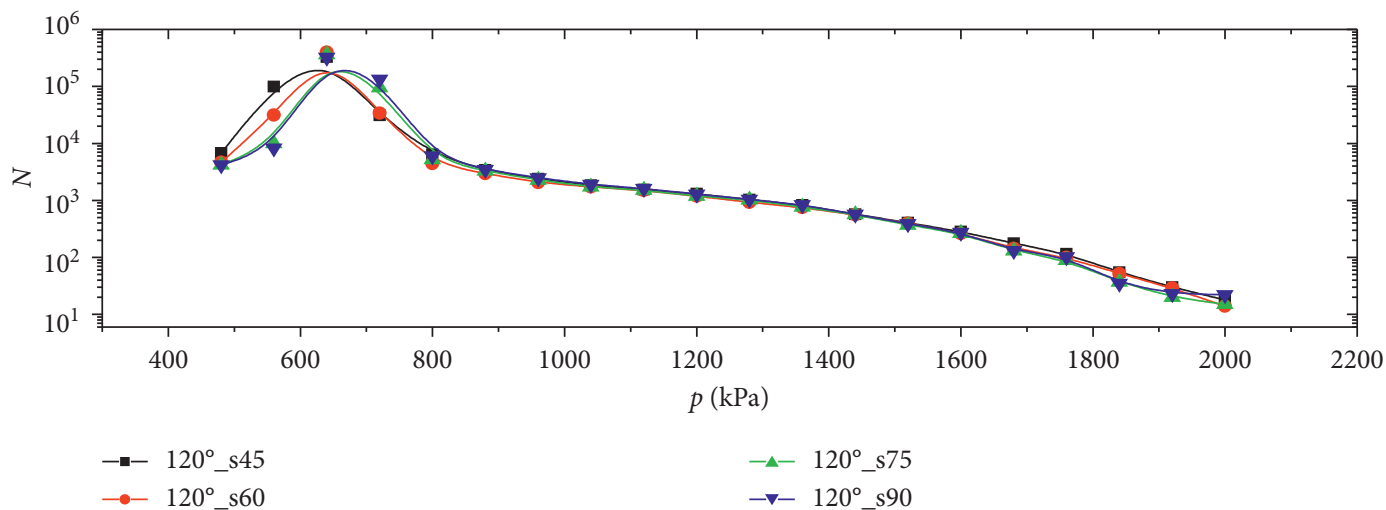

(c)

FIGURe 13: Number density versus peak height with different stand-off distances $(\sigma=0.0124)$.

distributed in an annular area on the impinged surface, and when the stand-off distance is increased, more area of the sensitive region of the sensor is exposed on the collapsing region. For the $40^{\circ}$ nozzle, the impact of the bubbles on the sensor increased obviously with the increase of the stand-off distance, while for the nozzle with larger divergent angle, the tendency is consistent, but the difference becomes smaller. For the nozzle with $120^{\circ}$ divergent angle, the number density curve for the impact load only changed slightly when the stand-off distance is increased. It can be inferred that the outlet geometry of the nozzle affects not only the cavitation generation but also the evolution and spatial distribution of the bubbles. For the nozzles with larger divergent angle, the velocity field diffuses faster as the main flow moves downstream which makes the bubbles distributed more evenly. For better application of the cavitation jet, the outlet shape of the nozzles as well as the stand-off distance from the target can be optimized according to the requirement of the application sites. 


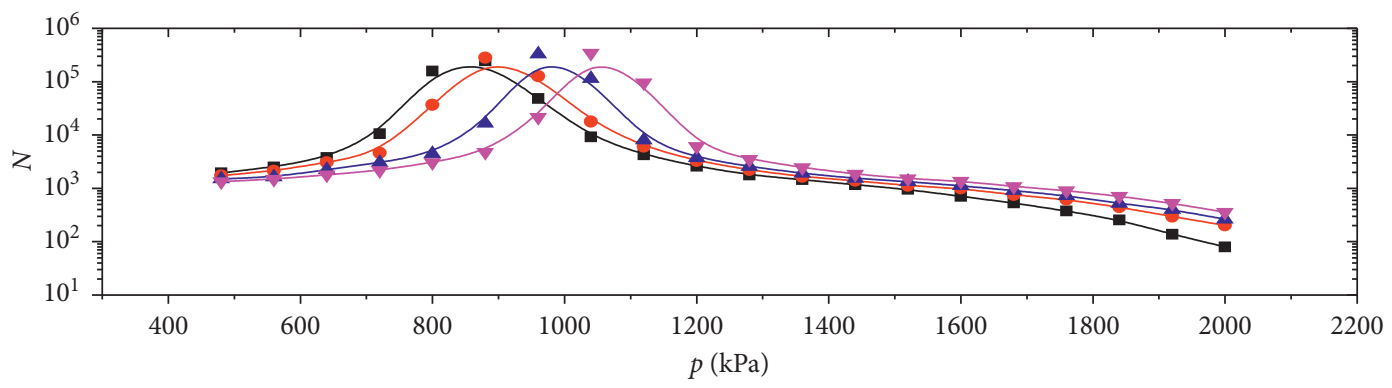

$$
\begin{aligned}
& \text { - } 40^{\circ} \text { s } 455 \text { - } 40^{\circ} \_ \text {s75 } \\
& \rightarrow 40^{\circ} \mathrm{s} 60 \quad \rightarrow 40^{\circ} \mathrm{s} 90
\end{aligned}
$$

(a)

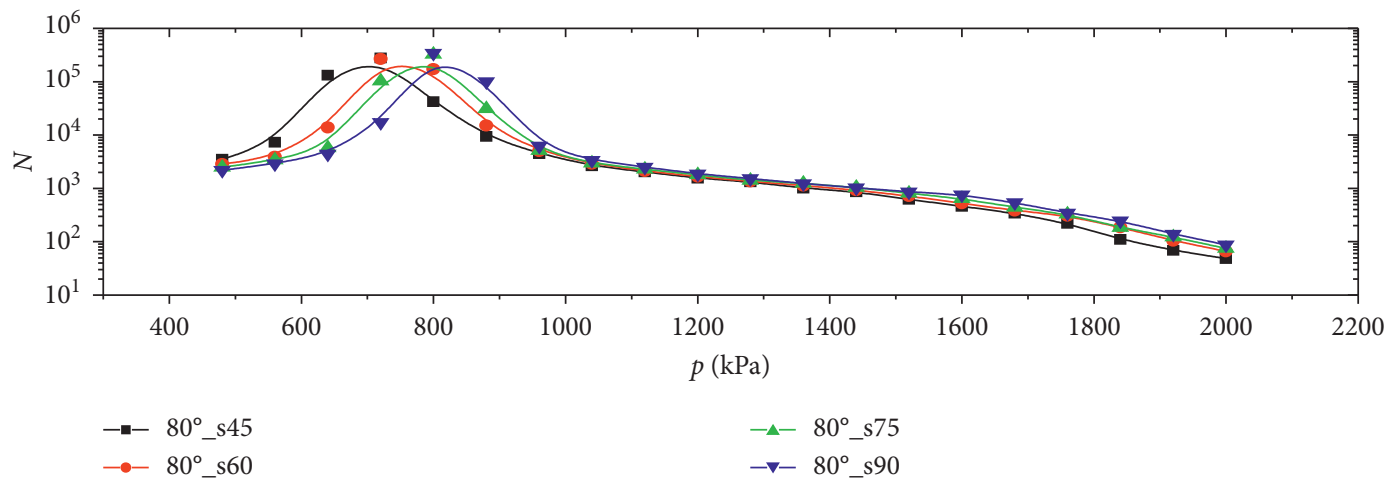

(b)

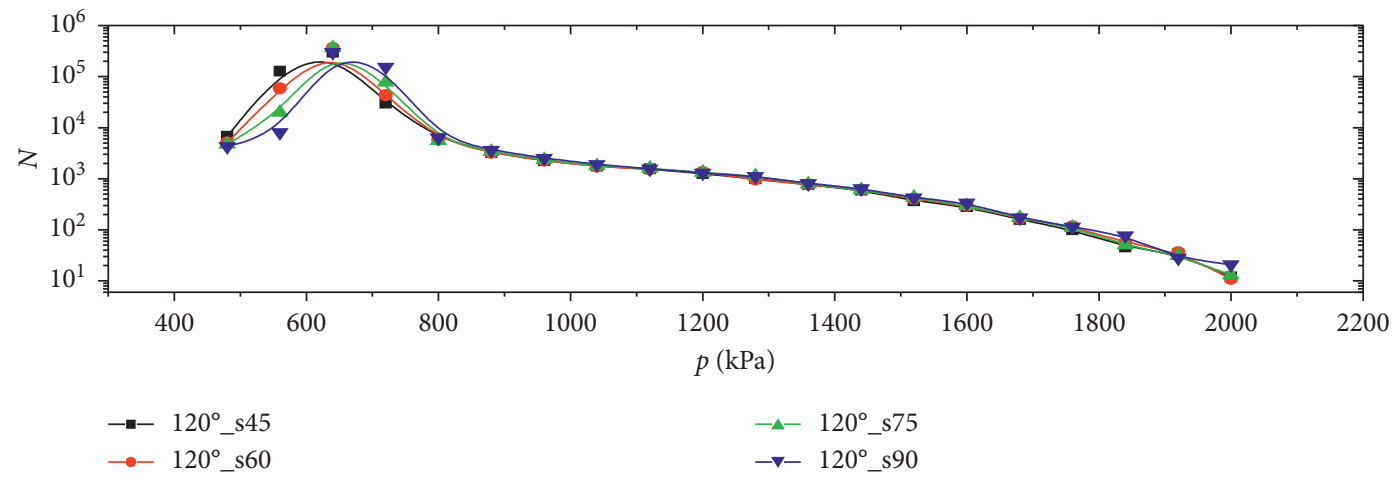

(c)

Figure 14: Number density versus peak height with different stand-off distances $(\sigma=0.0099)$.

\section{Conclusions}

In the current research, the unsteady characteristics of the high-pressure submerged cavitation jet are investigated using high-speed photography. The growing, shedding, and collapse period of the cavitation bubbles are observed. The grayscale image analysis method is used to correlate the cavitation concentration with the image grayscale. The influence of pumping pressure and angle of nozzle outlet expansion on the strength, stability, and unsteady characteristics of cavitation jets was studied. The research results are as follows:

(1) The cavitation number has a great influence on the intensity of bubble distribution. As the pump pressure increases, the length, width, and concentration of the cavitation cloud increase significantly, the position where the cavitation cloud breaks moves downstream, and the frequency for development and collapse of cavitation has dropped significantly. According to FDM analysis, at different pump pressures, the location of the collapse occurs mainly at the rear end of the bubble cloud in the downstream section after the shedding.

(2) The outlet divergent angle of the convergent-divergent-type nozzle also has an influence on the development of the bubble. For the nozzle of smaller divergent angle, the cavitation jet with higher cavitation concentration can be obtained, but the bubble 
distribution generated by the nozzle is only concentrated in a small region of the nozzle outlet. For the large divergence angle nozzle, the cavitation ability is greatly weakened due to insufficient binding force to the outflow at the nozzle outlet. The cavitation intensity is low and the distribution range is relatively small. Finally, it is found that there exists an optimal value for the divergent angle between $40^{\circ}$ and $120^{\circ}$, so that the cavitation concentration of the submerged cavitation jet can reach the highest level and the distribution region of cavitation is wide.

(3) The cavitation cloud generated by the high-pressure submerged jet inevitably has a shedding phenomenon. In each shedding period, the cavitation cloud undergoes three processes of growth, shedding, and collapse. The frequency of this process is between $0.5 \mathrm{kHz}$ and $2 \mathrm{kHz}$. It is much higher than the alternating current frequency of the halogen lamp light source and the moving frequency of the pump plunger. It can be judged that the frequency is related to the pressure fluctuation of the flooding jet and the self-oscillation of the fluid as it passes through the nozzle.

(4) The impact load of the cavitation jet is mainly affected by the stand-off distance of the nozzle from the impinged target, while the nozzle outlet geometry also has an effect on the impact performance. The number distribution of the impact peaks on the impinged surface caused by the bubble collapse near the central area of the jet has a Gaussian distribution and the peaks are in the range between $600 \mathrm{kPa}$ and $1200 \mathrm{kPa}$. The performance of the cavitation jet can be improved by optimizing the stand-off distance and the outlet geometry of the nozzles.

\section{Data Availability}

The data used to support the findings of this study are included within the article.

\section{Conflicts of Interest}

The authors declare that they have no conflicts of interest regarding the publication of this work.

\section{Acknowledgments}

This research was funded by the National Natural Science Foundation of China (nos. 51679111, 51409127, and 51579118), National Key R\&D Program Project (no. 2017YFC0403703), PAPD, Six Talents Peak Project of Jiangsu Province (no. HYZB-002), Key R\&D Program Project in Jiangsu Province (nos. BE2017126 and BE2016319), the Natural Science Foundation of Jiangsu Province (nos. BK20161472 and BK20160521), Science and Technology Support Program of Changzhou (no. CE20162004), Key R\&D Program Project of Zhenjiang (no. SH2017049), and the Scientific Research Start Foundation Project of Jiangsu University (no. 13JDG105).

\section{References}

[1] W. J. Kogelmann, E. D. Thimons, J. E. Virgona, and L. A. Weakly, "Water-jet-assisted mechanized oil shale mining factory test," in Proceedings of the Oil Shale Symposium, pp. 56-77, Colorado School of Mines, Golden, CO, USA, 1989.

[2] L. Sun, Y. J. Gong, Z. M. Zhang, and Z. W. Wang, "Software technology research on control system of ultra high pressure water jet rust removal equipment," Advanced Materials Research, vol. 721, pp. 372-376, 2013.

[3] D. Arola, A. E. Alade, and W. Weber, "Improving fatigue strength of metals using abrasive waterjet peening," $M a$ chining Science and Technology, vol. 10, no. 2, pp. 197-218, 2006.

[4] C. Lei, J. Guan, Y. Yu et al., "Study on the inner flow field of artificial submerged cavitating water jet nozzle," Shandong Chemical Industry, vol. 45, no. 11, pp. 144-146, 2016.

[5] A. Momma and A. Lichtarowicz, "A study of pressures and erosion produced by collapsing cavitation," Wear, vol. 186187, no. 2, pp. 425-436, 1995.

[6] T. Okada, Y. Iwai, S. Hattori et al., "Relation between impact load and the damage produced by cavitation bubble collapse," Wear, vol. 184, no. 2, p. 152, 1995.

[7] L. Bai, L. Zhou, X. Jiang, Q. Pang, and D. Ye, "Vibration in a multistage centrifugal pump under varied conditions," Shock and Vibration, vol. 2019, no. 3, pp. 1-9, 2019.

[8] Y. C. Wang and Y. W. Chen, "Application of piezoelectric PVDF film to the measurement of impulsive forces generated by cavitation bubble collapse near a solid boundary," $E x$ perimental Thermal \& Fluid Science, vol. 32, no. 2, pp. 403414, 2008.

[9] W. Li, L. Ji, W. Shi, L. Zhou, X. Jiang, and Y. Zhang, "Fluidstructure interaction study of a mixed-flow pump impeller during startup," Engineering Computations, vol. 35, no. 1, pp. 18-34, 2018.

[10] L. Ji, W. Li, W. Shi, H. Chang, and Z. Yang, "Energy characteristics of mixed-flow pump under different tip clearances based on entropy production analysis," Energy, vol. 199, p. 117447, 2020.

[11] X.-W. Luo, Y. Ji, and Y. Tsujimoto, "A review of cavitation in hydraulic machinery," Journal of Hydrodynamics, vol. 28, no. 3, pp. 335-358, 2016.

[12] H. Xu, X. Luo, and X. Peng, "Numerical analysis of cavitation evolution and excited pressure fluctuation around a propeller in non-uniform wake," International Journal of Multiphase Flow, vol. 43, pp. 13-21, 2012.

[13] N. Fujisawa, Y. Fujita, K. Yanagisawa et al., "Simultaneous observation of cavitation collapse and shock wave formation in cavitating jet," Experimental Thermal \& Fluid Science, vol. 94, pp. 159-167, 2018.

[14] H. Soyama, T. Kusaka, and M. Saka, "Peening by the use of cavitation impacts for the improvement of fatigue strength," Journal of Materials Science Letters, vol. 20, no. 13, pp. 1263-1265, 2001.

[15] H. Soyama and M. Asahara, "Improvement of the corrosion resistance of a carbon steel surface by a cavitating jet," Journal of Materials Science Letters, vol. 19, no. 13, pp. 1201-1205, 2000.

[16] Y. Yamauchi, H. Soyama, Y. Adachi et al., "Suitable region of high-speed submerged water jets for cutting and peening," International Journal of Multiphase Flow, vol. 22, no. 22, pp. 31-38, 2008. 
[17] L. Gensheng, S. Zhonghou, Z. Changshan, Z. Debin, and C. Hongbing, "Investigation and application of self-resonating cavitating water jet in petroleum engineering," Issues in Mental Health Nursing, vol. 23, no. 1, pp. 1-15, 2005.

[18] O. M. Dan and H. Soyama, "Optimization of cavitation peening parameters for fatigue performance of carburized steel using taguchi methods," Journal of Materials Processing Technology, vol. 178, no. 1, pp. 234-240, 2006.

[19] Z. Fang, Y. Kang, and X. Wang, "Effects of nozzle inner surface roughness on the cavitation erosion characteristics of high speed submerged jets," Experimental Thermal and Fluid Science, vol. 74, pp. 444-452, 2016.

[20] S. Hattori, T. Hirose, and K. Sugiyama, "Prediction method for cavitation erosion based on measurement of bubble collapse impact loads," Wear, vol. 269, no. 7, pp. 507-514, 2009.

[21] S. Hattori, Y. Goto, T. Fukuyama et al., "Influence of temperature on erosion by a cavitating liquid jet," Wear, vol. 260, no. 11-12, pp. 1217-1223, 2006.

[22] B. S. Mann, V. Arya, and B. K. Pant, "Cavitation erosion behavior of HPDL-treated TWAS-coated Ti6Al4V alloy and its similarity with water droplet erosion," Journal of Materials Engineering and Performance, vol. 21, no. 6, pp. 849-853, 2012.

[23] S. Hattori, T. Ogiso, Y. Minami, and I. Yamada, "Formation and progression of cavitation erosion surface for long exposure," Wear, vol. 265, no. 11-12, pp. 1619-1625, 2008.

[24] H. Soyama, Y. Yanauchi, K. Sato, T. Ikohagi, R. Oba, and R. Oshima, "High-speed observation of ultrahigh-speed submerged water jets," Experimental Thermal and Fluid Science, vol. 12, no. 4, pp. 411-416, 1996.

[25] G. Peng, A. Wakui, Y. Oguma, S. Shimizu, and H. Ji, "Periodic behavior of cavitation cloud shedding in submerged water jets issuing from a sheathed pipe nozzle," Journal of Flow Control, Measurement \& Visualization, vol. 06, no. 01, pp. 15-26, 2018.

[26] K. Sato, Y. Taguchi, and S. Hayashi, "High speed observation of periodic cavity behavior in a convergent-divergent nozzle for cavitating water jet," Journal of Flow Control, Measurement \& Visualization, vol. 1, no. 3, pp. 102-107, 2013.

[27] E. A. F. Hutli and M. S. Nedeljkovic, "Formula for upstream pressure, nozzle geometry and frequency correlation in shedding/discharging cavitation clouds determined by visualization of submerged cavitating jet," in Proceedings of the International Conference on Fluid Mechanics, Springer, Shanghai, China, pp. 1978-1980, July 2007.

[28] R. Watanabe, T. Kikuchi, T. Yamagata, and N. Fujisawa, "Shadowgraph imaging of cavitating jet," Journal of Flow Control, Measurement \& Visualization, vol. 3, no. 3, pp. 106-110, 2015.

[29] J. P. Franc, M. Riondet, A. Karimi et al., "Impact load measurements in an erosive cavitating flow," Journal of Fluids Engineering, vol. 133, no. 12, p. 121301, 2011. 